\title{
Large-Scale Surveys of Pulsating Stars for Studying Stellar Populations in the Inner Galaxy
}

\author{
Noriyuki Matsunaga ${ }^{1}$ \\ ${ }^{1}$ Department of Astronomy, The University of Tokyo, 7-3-1 Hongo, Bunkyo-ku, Tokyo \\ 113-0033, Japan \\ email: matsunaga@astron.s.u-tokyo.ac.jp
}

\begin{abstract}
Surveys of pulsating stars in the inner Galaxy have been very limited, but recent large-scale surveys are rapidly bringing us new samples of various kinds of variable stars and new insights into stellar populations therein. Because of the severe interstellar extinction along the Galactic disc, the stellar populations in the inner Galaxy are more easily observed in the infrared, but even in the infrared the interstellar extinction may cause a serious problem in revealing their accurate characteristics. Here we review recent discoveries of Cepheids and Miras, two kinds of luminous pulsating stars with period-luminosity relation, in the inner Galaxy.
\end{abstract}

Keywords. stars: AGB and post-AGB, stars: carbon, stars: distances, (stars: variables:) Cepheids, stars: variables: other, (ISM:) dust, extinction, Galaxy: bulge, Galaxy: disc, Galaxy: stellar content, Galaxy: structure

\section{Introduction}

Classical pulsating stars such as Cepheids and Miras are useful tracers of the Galaxy. Period-luminosity relations of those objects allow us to determine the distances to individual stars (see Matsunaga 2017, and references therein). Each group of pulsating stars has a typical range of ages, so that we can learn ages of stellar populations in galaxies based on pulsating stars. Classical Cepheids are young, Miras are intermediate to old, and type II Cepheids and RR Lyrs are all old. Especially, ages of classical Cepheids are anti-correlated with pulsation periods, and that makes it possible to estimate their accurate ages (Bono et al. 2005). Furthermore, when appropriate datasets available, we can combine kinetic and chemical parameters of these objects to reconstruct chemodynamical information for tracing the structure and evolution of the Galaxy.

There are some complications which should be borne in mind when pulsating stars are used as tracers of the Galaxy. Firstly, classification of the variability type is not always easy and should be based on good data. For example, it is possible to get confused between pulsating stars, eclipsing binaries, and objects with bright or dim stellar spots; see, e.g., a controversy about faint objects towards the Galactic disc found in Chakrabarti et al. (2015, 2017) and Pietrukowicz et al. (2015b). Secondly, stellar rotation may affect ages of Cepheids and maybe other stars as discussed by Anderson et al. (2014, 2016). Thirdly, evolution in binary systems complicates the implication of pulsating stars concerning stellar populations. The ages of such objects can be totally different from those of isolated stars but with similar variable characteristics. For example, Feast, Menzies, \& Whitelock (2013) found a carbon-rich Mira in an old-aged globular clusters. Such old stars are not expected to evolve into carbon stars but a merged star can become a carbon star. Such objects must be relatively small in number, but when we discuss rare objects we need to take such evolutionary paths into consideration. We'll come back to this point later. 
Another interesting case of unusual pulsating stars evolved in a binary system is found in Pietrzyński et al. (2012). While these complications require further investigations to address their impacts and necessary corrections, if possible, on studies on the Galaxy, at least a large fraction of pulsating stars are useful for studying stellar populations and their distributions in the Galaxy.

While pulsating stars have been actively used for studying the Galactic halo (e.g. Huxor \& Grevel 2015; Fiorentino et al. 2017), our focus in this review is on the inner part of the Galaxy. The use of pulsating stars as tracers of the inner part and the disc of the Galaxy has been limited by the incompleteness of surveys (e.g. see figure 1 of Matsunaga 2012). The main reasons for the incompleteness are twofold: (1) early surveys were optical and relatively shallow and thus were largely affected by the interstellar extinction in the disc region, and (2) sky coverage of sufficiently deep recent surveys, in particular those in the infrared, has been limited. In terms of the sky coverage, Gaia will produce an unprecedented catalogue of pulsating stars in the entire range of the Galaxy, although the interstellar extinction limits its reach across the Galactic plane. Windmark, Lindegren, \& Hobbs (2011) predicted that the total number of Cepheids in the Galaxy is roughly 20,000 based on a simple exponential-disc model and about half of them would be detected by Gaia. There will be many regions where Cepheids are too much obscured for Gaia; in such obscured regions, one cannot expect to get stellar distribution based on Gaia's parallaxes, and pulsating stars found in the infrared will be crucial even in the Gaia era.

In this review, we summarize the currently known samples of Cepheids and Miras in the inner Galaxy in the sections 2 and 3, respectively. Fig. 1 summarizes currently known Cepheids and Miras towards the Galactic bulge. Unless otherwise mentioned, our discussions are limited to this range of the sky. At the distance to the Galactic centre, $2.3 \mathrm{kpc}$ (de Grijs \& Bono 2016), the horizontal stretch of Fig.11, 27 degrees, corresponds to approximately $4 \mathrm{kpc}$. The dominant component in this range, especially in terms of stellar mass $\left(2 \times 10^{10} \mathrm{M}_{\odot}\right.$, Valenti et al. 2016), is the bulge with the bar structure inclined by 25-30 degrees to the direction of the Sun (Bland-Hawthorn \& Gerhard 2016). However, several systems with different characteristics are overlapped in this range, which sometimes complicates interpretation of observation data on objects in this direction. At the very centre exists the Nuclear Stellar Cluster surrounding the supermassive blackhole, and this cluster is within the Nuclear Stellar Disc (or also known as the Central Molecular Zone). The former has $\sim 2 \times 10^{7} \mathrm{M}_{\odot}$ in stars within the radius of $10 \mathrm{pc}$, and the latter has $\sim 10^{9} \mathrm{M}_{\odot}$, within the radius of $200 \mathrm{pc}$, of which a few percent are found in interstellar gas and dust (Launhardt, Zylka, \& Mezger). The size of the NSD is illustrated by the ellipse in Fig. 1. In the foreground and background extended is the Galactic disc. These different systems host different stellar populations and thus different sets of pulsating stars as we see below.

RR Lyrs and type II Cepheids are useful tracers of old stellar population and have been found in the bulge, although they are outside the scope of this review. Readers are referred to recent observational results in the following papers: Pietrukowicz et al. (2012,2015), Dékány et al. (2013), Gran et al. (2015, 2016), Minniti et al. (2016), and Dong et al. (2017) concerning RR Lyrs, and Soszyński et al. (2011), Matsunaga et al. (2013), and Bhardwaj et al. (2017) concerning type II Cepheids. The surveys are rather incomplete for these important types of pulsating stars (see, e.g. figure 2 in Pietrukowicz et al. 2015 and figure 6 in Soszyński et al. 2011). 

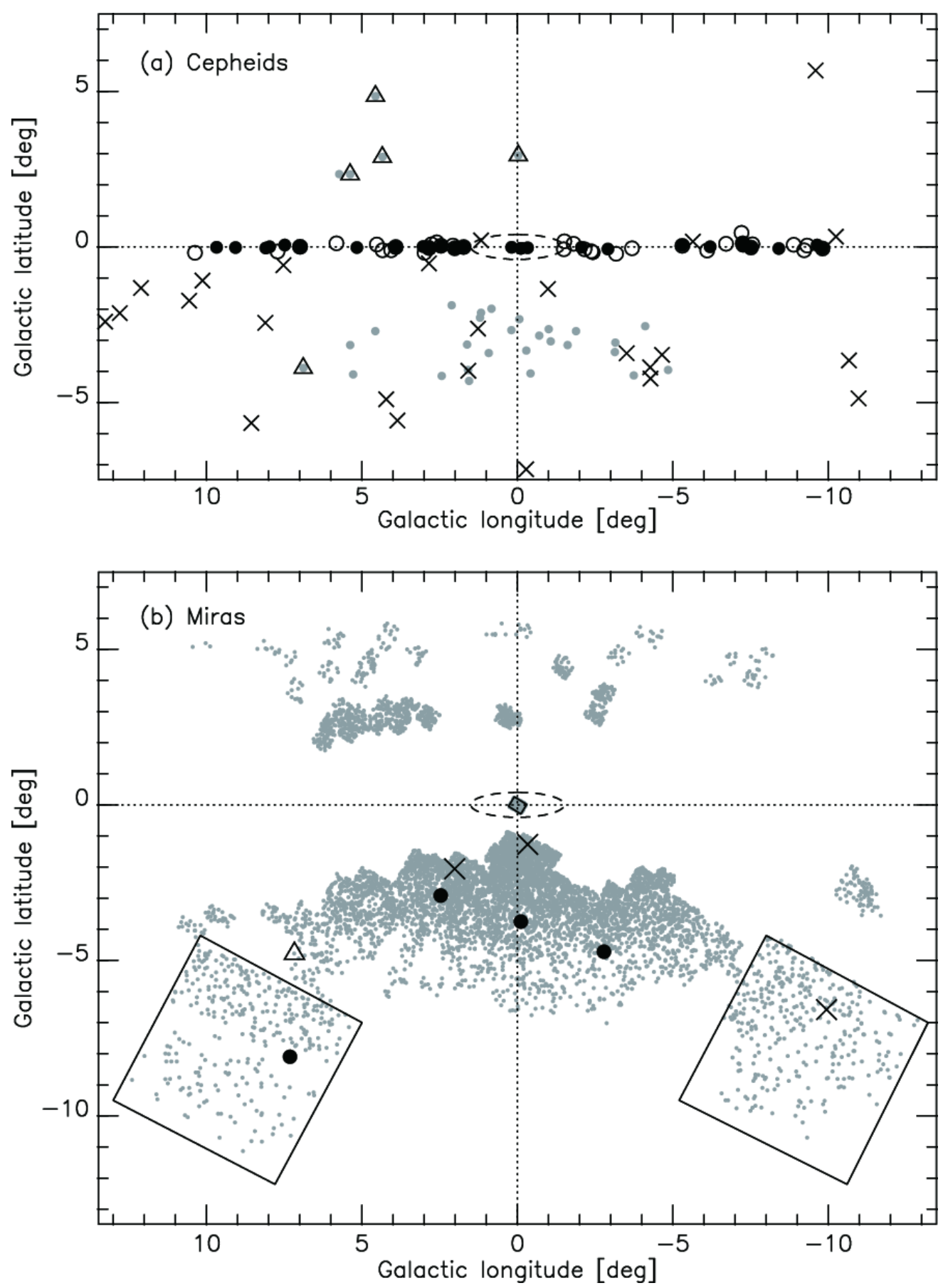

Figure 1. Distributions of known Cepheids (panel a) and Miras (panel b). The dashed ellipse around $(l, b)=(0,0)$ suggests the approximate range of the Nuclear Stellar Disk (Launhardt et al. 2002). The objects included in each panel are as follows: (a) Grey dots indicate classical Cepheids from OGLE (Soszyński et al. 2011), among which five indicated by triangles are discussed in Feast et al. (2014) Open and filled circles indicate those found by Dékány et al. (2015ab) and by Matsunaga et al. (2016), respectively. Within the dashed ellipse for the NSD located are four Cepheids discussed in Matsunaga et al. (2011, 2015). Cepheids in this region in the catalogue of Fernie et al. (1995) are indicated by crosses, but they are all located within $3 \mathrm{kpc}$ of the Sun and are foreground objects. (b) Grey dots indicate Miras from OGLE (Soszyński et al. 2013) and those discussed in Catchpole et al. (2016) whose main targets are located within the two rectangles. Four filled circles indicate the carbon-rich Miras reported in Matsunaga et al. (2017), while the cross and triangle symbols indicate those which are probably foreground and background of the bulge as discussed in the same paper. 


\section{Cepheids towards the inner Galaxy}

Classical Cepheids are pulsating supergiants with pulsation periods ranging from one day to $\sim 80$ days or even longer. They are 10-300 Myr old and their initial masses are $4-10 \mathrm{M}_{\odot}$ (Bono et al. 2005). Their period-luminosity relation is one of the most important steps of the cosmic distance ladder (Freedman et al. 2001; Riess et al. 2016). Since the pioneering work by Baade (summarized in Baade 1956), classical Cepheids are distinguished from type II Cepheids which are evolved low-mass stars aged 10 Gyr. We'll consider only classical Cepheids hereinafter.

Classical Cepheids, as representatives of young stars, have been used for studying various characteristics of the Galactic disc (e.g. Feast \& Whitelock 1997; Majaess, Turner, \& Lane 2009). In particular, Cepheids have been the most successful tracers of the metallicity gradient, i.e. how the metallicity changes as a function of the distance from the Galactic centre (Genovali et al. 2014, 2015; da Silva et al. 2016). However, the previous survey of classical Cepheids is incomplete as we discussed above. Fig. 11 plots about a dozen of previously known Cepheids in the DDO database (Fernie et al. 1995), but they are all within $3 \mathrm{kpc}$ of the Sun. It is considered that the bulge is dominated by old stellar populations even if a small fraction of young stars exist (Zoccali et al. 2003; Clarkson et al. 2011; Benzby et al. 2013), and therefore classical Cepheids are not expected and haven't been found in the bulge. In contrast, the NSD is known to host stars with a wide range of ages (Serabyn, \& Morris 1996; Figer et al. 2004). Matsunaga et al. $(2011,2015)$ in fact found four classical Cepheids in this system. They are located within 0.35 degrees of the Galactic centre, or within $50 \mathrm{pc}$ (projected distance), and their radial velocities are consistent with the rotation of the NSD (Matsunaga et al. 2015). Interestingly, all of them have periods around 20 days (18.8-23.5 days), which is significantly longer than the median period, $\sim 5$ days, of Galactic Cepheids, and thus are similarly aged $\sim 25 \mathrm{Myr}$, which puts an important constraint on the star formation history in this system (Matsunaga et al. 2011). The foreground interstellar extinction for these Cepheids are $\sim 2.5$ mag or more at around $2 \mu \mathrm{m}$, corresponding to $\sim 30$ mag in the optical, so that infrared observations are required for both surveys and follow-up studies.

Later, infrared surveys by Dekany et al. (2015ab) and Matsunaga et al. (2016) found dozens of Cepheids in the mid plane of the Galactic disc, i.e. nearly 0 degree in Galactic latitude (Fig.11). While Dékány et al. (2015b) suggested the presence of an inner thin disc of young stars surrounding the NSD, |Matsunaga et al. (2016)|reported the lack of young stars in the inner $2.5 \mathrm{kpc}$ except the NSD. As discussed in Matsunaga et al. (2016), the distances to these reddened Cepheids $\left(E_{H-K_{\mathrm{s}}}=1.2-2.5 \mathrm{mag}\right)$ have large errors due to the uncertainty in the extinction law, i.e. the wavelength dependency of the interstellar extinction. The four classical Cepheids in the NSD give an important constraint on the extinction law because they should be located at the distance of the Galactic centre regardless of the extinction. This supports the extinction law of Nishiyama et al. (2006) rather than that of Nishiyama et al. (2009) which was used by Dekany et al. (2015ab). More detailed discussions on the problem of the extinction law are found in the original paper of Matsunaga et al. (2016) and a recent review (Matsunaga 2017). The lack of Cepheids and young stars in the innermost part of the Galactic disc is supported by the distribution of observed H II regions and massive star-forming regions (e.g. Jones et al. 2013; Sanna et al. 2014). This suggests that there is no simple exponential disc extending into the centre in contrast to the model used by Windmark, Lindegren, \& Hobbs (2011), if we consider young stars, and we need further infrared surveys to map this region including the entire NSD and the interface between the discs and the bulge.

Another interesting sample of classical Cepheids towards the region of our interest but 
a few degrees away from the Galactic mid plane came from a large-scale optical survey, Optical Gravitational Lensing Experiment (OGLE). Soszyński et al. (2011) discovered 32 classical Cepheids towards the bulge (Fig.1) besides hundreds of type II Cepheids. Although most of the type II Cepheids belong to the bulge considering their positions on the period-Wesenheit diagram (figure 7 in Soszyński et al. 2011), most of the classical Cepheids seem to be located further than the bulge (their magnitudes are similar to those of type II Cepheids in spite of the large difference between intrinsic magnitudes for these two types). Five of the 32 classical Cepheids, triangles in the panel (a) of Fig.1. were investigated by Feast et al. (2014) in more detail, and their distances are larger than $20 \mathrm{kpc}$, indeed further than the bulge. Moreover, they are separated from the Galactic plane by $\sim 1 \mathrm{kpc}$ or more and belong to the flared part of the disc outskirts. Other Cepheids in Soszyński et al. (2011) are also located at similarly large distances and exotic objects like these five, but their nature needs to be revealed by further investigations.

\section{Miras towards the inner Galaxy}

Miras are pulsating giants with periods longer than 100 days. Their initial masses and ages are relatively wide: $1-9 \mathrm{M}_{\odot}$ corresponding to between $\sim 10 \mathrm{Gyr}$ and $30 \mathrm{Myr}$ (Iben 1983). There is an anti-correlation between periods and ages (Feast, Whitelock, \& Menzies 2006), although its relation is not so tight or established as the period-age relation of classical Cepheids. Nevertheless, a wide range of the ages of Miras is useful for tracing old to intermediate-age stellar populations. Miras also have the period-luminosity relation and serves as distance indicator, although one can find useful relations only in the infrared or the bolometric magnitude but not in the optical (Glass \& Lloyd Evans 1981; Feast et al. 1989; Whitelock et al. 2008). As has been discussed in Feast, \& Whitelock (2014) and Whitelock, \& Feast (2014), Miras are very bright in the infrared and can be a good alternative to Cepheids as distance indicators to galaxies at large distances for infrared facilities like James Webb Space Telescope (JWST) in the future.

Because of the high luminosities of Miras, surveys of Miras in the bulge, especially towards low-extinction windows, have been done from the early days (see the review given by Catchpole et al. 2016). Recently, Soszyński et al. (2013) found more than 6500 Miras using the OGLE dataset (Fig.1), which doesn't include the low-latitude region $\left(|b|<1^{\circ}\right)$. Such central parts require infrared surveys even if they are relatively shallow, and in fact early surveys in the near infrared already detected dozens or hundreds of Miras (Glass et al. 2001, and references therein) within the NSD. Matsunaga et al. (2009) detected over 500 Miras (with periods determined) and obtained the distance to the Galactic centre, $8.24 \mathrm{kpc}$, making use of multi-band photometry in $J H K_{\mathrm{s}}$ bands which was necessary to make correction of the interstellar extinction. This estimate or any other estimate based on standard candles (i.e. luminosity based distance indicators) is affected by the uncertainty in the extinction law described above (Matsunaga 2013). Even with this uncertainty in the distances, it is clear that Miras show a concentration towards the Galactic centre (Matsunaga et al. 2009; see also the large-scale density gradient in Fig. 1). It is, however, unclear how Miras in the central $100 \mathrm{pc}$ should be separated into the NSD and the extended bar-like bulge.

An important group of objects related to Miras, with at least some overlaps, is sources with maser emissions with $\mathrm{SiO}, \mathrm{H}_{2} \mathrm{O}$, and $\mathrm{OH}$ molecules. These emissions are produced in evolved stars with thick circumstellar shell, and those objects are often found to be Miras or related pulsating stars like semi-regulars (e.g. see the review by Habing 1996). In particular, $\mathrm{OH} / \mathrm{IR}$ stars characterized by $\mathrm{OH}$ maser emission and high infrared luminosity have played an important role in studying intermediate-age populations in the 
inner Galaxy (Lindqvist, Habing, \& Winnberg 1992; Wood, Habing, \& McGregor 1998). Their kinematics supports that these intermediate-age objects belong to the NSD. Later, Deguchi et al. (2004) detected $\mathrm{SiO}$ maser in 180 Miras of the catalogue published by Glass et al. (2001), and also found the rotation comparable to that of OH/IR stars. This seems to suggest that at least a part of the Miras near the centre belong to the NSD, but the more detailed studies on distributions of these Miras and maser sources should be done with more comprehensive datasets including kinematic information from both radial velocities and proper motions.

The recent discovery by Matsunaga et al. (2017) has revealed a rare group of objects, carbon-rich Miras, among the Miras in the bulge. They selected several candidates of carbon-rich Miras, among the Miras in Soszyński et al. (2013) and Catchpole et al. (2016) based on the $\left(J-K_{\mathrm{s}}\right)-([9]-[18])$ diagram in which one can distinguish objects with carbon-rich dust shell from those with oxygen-rich dust shell (Ishihara et al. 2011). Then, with spectroscopic follow-up observations, they confirmed 8 carbon-rich Miras of which 3 are foreground objects. These carbon-rich Miras are considered to represent relatively (or totally) unexplored stellar population(s). Both age and metallicity affect whether an AGB star is evolved into carbon-rich stars or not, and intermediate-age stars (a few Gyr) tend to finish the AGB phase as carbon stars (Marigo 2013). For the Galactic bulge, no carbon-rich AGB stars had been confirmed before. This was naturally understood because the dominant population of the bulge is old and metal-rich. Moreover, many stars are expected to be oxygen enhanced which makes it even harder to produce carbon-rich stars. Unfortunately, the ages and the origin of the carbon-rich Miras found in the bulge are still unclear because mass-augmented stars may evolve into carbon-rich stars even if they are old (Feast et al. 2013). Kinematics and chemical information may help uncover their origins.

\section{Summary}

In the last decade, large-scale surveys like OGLE and some near-infrared ones have revealed large collections of new Cepheids and Miras. The same is true for RR Lyrs and other kinds of variable stars. Nevertheless, Fig. 1 clearly shows that we still miss a large numbers of these objects particularly in the low-latitude region, in which every system of the inner Galaxy is overlapped (i.e. the Galactic bulge, the Nuclear Stellar Disk, and the Nuclear Stellar Clusters) together with the Galactic disc in the foreground and background. Several large-scale surveys including OGLE, VISTA Via Lactea (Minniti et al. 2010), and Gaia are expected to provide us with more complete samples of pulsating stars which will be crucial for studying stellar populations in the future. Various parameters are readily accessible using the period-luminosity and period-age relations, for example, and other observables for the pulsating stars, and they will remain to be useful tracers of stellar populations in the Galaxy.

\section{Acknowledgement}

The author appreciate financial support from the Japan Society for the Promotion of Science (JSPS) through the Grant-in-Aid, No. 26287028.

\section{References}

Anderson, R.I., Ekström, S., Georgy, C., Meynet, G., Mowlavi, N., Eyer, L. 2014, A\&A 564, A100

Anderson, R.I., Saio, H., Ekström, S., Georgy, C., Meynet, G. 2016, A\& A 591, A8 
Alonso-García, J., Dékány, I., Catelan, M., Contreras Ramos, R., Gran, F., Amigo, P., Leyton, P., \& Minniti, D. 2015, AJ 149, 99

Baade, W. 1956, PASP 68, 5

Bensby, T., et al. 2013, A\&A 549, A147

Bhardwaj, A., et al. 2017, A\&A, in press (arXiv:1707.03755)

Bland-Hawthorn, J., \& Gerhard, O. 2016, ARA\&A 54, 529

Bono, G., Marconi, M., Cassisi, S., Caputo, F., Gieren, W., \& Pietrzynski, G. 2005, ApJ 621, 966

Catchpole, R.M., Whitelock, P.A., Feast, M.W., Hughes, S.M.G., Irwin, M., Alard, C. 2016, MNRAS 455, 2216

Chakrabarti, S., Saito, R., Quillen, A., Gran, F., Klein, C., \& Blitz, L. 2015, ApJ (Letters) 802, L4

Chakrabarti, S., et al. 2017, ApJ 844, 159

Clarkson, W.I., et al. 2011, ApJ 735, 37

da Silva, R., et al. 2016, A\&A A 586, A125

de Grijs, R., \& Bono, G. 2016, ApJS 227, 5

Deguchi, S., et al. 2004, PASJ 56, 261

Dékány, I., Minniti, D., Catelan, M., Zoccali, M., Saito, R.K., Hempel, M., Gonzalez, O.A. 2013, ApJ (Letters) 776, L19

Dékány, I., et al. 2015a, ApJ (Letters) 799, L11

Dékány, I., et al. 2015b, ApJ (Letters) 812, L29

Dong, H., et al. 2017, MNRAS 471, 3617

Feast, M.W., Glass, I.S., Whitelock, P.A., \& Catchpole, R.M. 1989, MNRAS 241, 375

Feast, M. W., \& Whitelock, P.A. 2014, IAUS 298, 40

Feast, M.W., \& Whitelock, P.A. 1997, MNRAS 291, 683

Feast, M.W., Whitelock, P.A., \& Menzies, J.W. 2006, MNRAS 369, 791

Feast, M.W., Menzies, J.W., \& Whitelock, P.A. 2013, MNRAS 428, L36

Feast, M.W., Menzies, J.W., Matsunaga, N., \& Whitelock, P.A. 2014, Nature 509, 342

Fernie, J.D., Evans, N.R., Beattie, B., \& Seager, S. 1995, Information Bulletin on Variable Stars 4148, 1

Figer, D.F., Rich, R.M., Kim, S.S., Morris, M., \& Serabyn, E. 2004, ApJ 601, 319

Fiorentino, G., et al. 2017, A\&A 599, A125

Freedman, W.L., et al. 2001, ApJ 553, 47

Genovali, K., et al. 2014, A\&A 566, A37

Genovali, K., et al. 2015, A\&A 580, A17

Glass, I.S., \& Lloyd Evans, T. 1981, Nature 291, 303

Glass, I.S., Matsumoto, S., Carter, B.S., \& Sekiguchi, K. 2001, MNRAS 321, 77

Gran, F., Minniti, D., Saito, R.K., Navarrete, C., Dékány, I., McDonald, I., Contrares, R.R., \& Catelan, M. 2015, A\&GA 575, A114

Gran, F., et al. 2016, AछA 591, A145

Habing H.J. 1996, $A \mathscr{E} A R$ 7, 97

Huxor, A.P., \& Grebel, E.K. 2015, MNRAS 453, 2653

Iben, I.Jr., \& Renzini, A. 1983, ARA\& $A$ 21, 271

Ishihara, D., Kaneda, H., Onaka, T., Ita, Y., Matsuura, M., \& Matsunaga, N. 2011, A\&A 534, A79

Jones, C., Dickey, J.M., Dawson, J.R., McClure-Griffiths, N.M., Anderson, L.D., \& Bania, T.M. 2013, ApJ 774, 117

Launhardt, R., Zylka, R., \& Mezger, P.G. 2002, A\&A 384, 112

Lindqvist, M., Habing, H.J., \& Winnberg, A. 1992, A\&A 259, 118

Majaess, D.J., Turner, D.G., \& Lane, D.J. 2009, MNRAS 398, 263

Marigo, P., Bressan, A., Nanni, A., Girardi, L., \& Pumo, M.L. 2013, MNRAS 434, 488

Matsunaga, N., Kawadu, T., Nishiyama, S., Nagayama, T., Hatano, H., Tamura, M., Glass, I. S., \& Nagata, T. 2009, MNRAS 399, 1709

Matsunaga, N., Kawadu, T., Nishiyama, S., et al. 2011, Nature 188, 477

Matsunaga, N. 2012, Journal of Physics: Conference Series 372, 12026 
Matsunaga, N. 2013, IAUS 289, 109

Matsunaga, N., Feast, M. W., Kawadu, T., et al. 2013, MNRAS 429, 385

Matsunaga, N., Fukue, K., Yamamoto, R., et al. 2015, ApJ 799, 46

Matsunaga, N., Feast, M. W., Bono, G., et al. 2016, MNRAS 462, 414

Matsunaga, N. 2017, EPJ Web of Conferences 152, 1007

Matsunaga, N., Menzies, J. W., Feast, M. W., Whitelock, P. W., Onozato, H., Barway, S., \& Aydi, E. 2017, MNRAS 469, 4949

Minniti, D., et al. 2010, New Astron. 15, 433

Minniti, D., Contreras Ramos, R., Zoccali, M., Rejkuba, M., Gonzalez, O.A., Valenti, E., \& Gran, F. 2016, ApJ (Letters) 830, L14

Nishiyama, S., et al. 2006, ApJ 638, 839

Nishiyama, S., Tamura, M., Hatano, H., Kato, D., Tanabé, T., Sugitani, K., Nagata, T. 2009, ApJ 696, 1407

Pietrukowicz, P., et al. 2012, ApJ 750, 169

Pietrukowicz, P., et al. 2015a, ApJ 811, 113

Pietrukowicz, P., et al. 2015b, ApJ (Letters) 813, L40

Pietrzyński, G., et al. 2012, Nature 484, 75

Riess, A.G., et al. 2016, ApJ 826, 56

Sanna, A., et al. 2014, ApJ 781, 108

Serabyn, E., \& Morris, M. 1996, Nature 382, 602

Soszyński, I., et al. 2011, AcA 61, 285

Soszyński, I., et al. 2013, AcA 63, 21

Valenti, E. et al. 2016, A\&A 587, L6

Whitelock, P.A., \& Feast, M. W. 2014, EAS Publications Series 67-68, 263

Whitelock, P.A., Feast, M.W., \& van Leeuwen, L. 2008, MNRAS 386, 313

Windmark, F., Lindegren, L., \& Hobbs, D. 2011, A\&A 530, A76

Wood, P.R., Habing, H.J., \& McGregor, P.J. 1998, A $\mathscr{E} A$ 336, 925

Zoccali, M. et al. 2003, A\&A 399, 931 